\title{
Virginia Brabender and April Fallon: Group Development in Practice: Guidance for Clinicians and Researchers on Stages and Dynamics of Change
}

\author{
American Psychological Association, 315 pages, \$59.95
}

\author{
Max Rosenbaum
}

Published online: 6 January 2010

(C) Springer Science+Business Media, LLC 2010

Brabender and Fallon believe that a "progressive stage or developmental approach to group psychotherapy has many benefits and can be applied by group therapists from very different theoretical perspectives." Brabender and Fallon note the bewildering amount of information that confronts the beginning group therapist, and their effort is admirable as they confront group psychotherapy, viewed as a clinical practice that remains in search of a theory.

In their effort to make sense of the "bewildering" amount of clinical data, Brabender and Fallon have ignored the different goals of group psychotherapists. By overlooking the early history of group psychotherapy, they have omitted some important pioneers in the field. As this reviewer pointed out more than fifty years ago, if psychotherapy is on a continuum of reparative to reconstructive, different group leaders have different therapeutic goals. The goal of a group leader who is wedded to an interactional approach (e.g., Yalom) is vastly different from a group psychotherapist who emphasizes the intrapsychic (e.g., Alexander Wolf- an early pioneer). Likewise, group therapy will be different when working in a mental hospital (e.g., Louis Wender), or leading groups of tubercular patients (e.g., Joseph Pratt). Brabender and Fallon appear to be resonant to Bion's approach to group treatment and note how much Melanie Klein influenced Bion (see his personal psychoanalysis with Klein).

The Wall Street Journal recently reported that "group therapy is at least $50 \%$ less expensive than individual therapy," (see Tuesday, March 24, 2009). Nonetheless, many practitioners believe in the value of therapy groups irrespective of the cost. Does the current book clarify important issues in group psychotherapy? The reviewer would say no. At times the writing is clear. At other times it seems disjointed. Brabender and Fallon should be commended for their efforts. Even if not "great", this book is a "good try."

M. Rosenbaum ( $\square)$

Nova Southeastern University, Davie, FL, USA

e-mail: bellemax@earthlink.net 